\title{
(2) OPEN ACCESS \\ Represent me: please! Towards an ethics of digital twins in medicine
}

\author{
Matthias Braun (ㄷ)
}

Correspondence to

Dr Matthias Braun, FAU,

Erlangen, Germany;

matthias.braun@fau.de

Received 7 February 2020

Revised 11 January 2021

Accepted 21 January 2021

Published Online First

15 March 2021

\section{Linked}

- http://dx.doi.org/10.1136/ medethics-2021-107447

- http://dx.doi.org/10.1136/ medethics-2021-107448

- http://dx.doi.org/10.1136/ medethics-2021-107449

- http://dx.doi.org/10.1136/ medethics-2021-107526

- http://dx.doi.org/10.1136/ medethics-2021-107460

- http://dx.doi.org/10.1136/ medethics-2021-107451

Check for updates

(c) Author(s) (or their employer(s)) 2021. Re-use permitted under CC BY-NC. No commercial re-use. See rights and permissions. Published by BMJ.

\begin{tabular}{|l|}
\hline To cite: Braun M. \\
J Med Ethics \\
2021;47:394-400.
\end{tabular}

\begin{abstract}
Simulations are used in very different contexts and for very different purposes. An emerging development is the possibility of using simulations to obtain a more or less representative reproduction of organs or even entire persons. Such simulations are framed and discussed using the term 'digital twin'. This paper unpacks and scrutinises the current use of such digital twins in medicine and the ideas embedded in this practice. First, the paper maps the different types of digital twins. A special focus is put on the concrete challenges inherent in the interactions between persons and their digital twin. Second, the paper addresses the questions of how far a digital twin can represent a person and what the consequences of this may be. Against the background of these two analytical steps, the paper defines first conditions for digital twins to take on an ethically justifiable form of representation.
\end{abstract}

\section{INTRODUCTION}

Imagine you have a serious illness, such as a severe heart defect. You are not in acute life-threatening danger, and from a medical perspective you are reasonably well adjusted, but it is foreseeable that surgery will be necessary. The risks of this surgery are difficult to predict, and the possible consequences of the operation are uncertain. Good advice on the matter is expensive.

One plan sounds bold: you will not be operated on at first, but a specific simulation of your heart will be created which can then be used to investigate possible difficulties and complications. In such a simulation, which will be referred to in the following as a 'digital twin', predictions could also be made about the potential course of the disease.

Just fiction? Maybe not. There are several ongoing projects and collaborations that aim to create digital twins. Initial studies demonstrate the potential for digital twins to allow clinicians to discover and predict problems before they manifest, to better prepare for surgeries and to conduct experiments with possible alternative treatments. ${ }^{1}$

${ }^{2}$ For example, a number of projects endeavour to create multiscale digital simulations of the heart to monitor circulation and to virtually test medication in development-ultimately to predict drug-induced arrhythmias. ${ }^{3}$ Furthermore, there are attempts to enable the digital twin to not only be a passive simulation but to interact with machines and tools or their representations. ${ }^{45}$ In this respect, there is the potential for digital twins to be a key technology in the future of healthcare, ${ }^{6}$ promising health improvements, the reduction of healthcare costs and increased personal freedom in dealing with one's biological conditions. ${ }^{7}$
Despite the question of which part of the digital twin scenario is already a real-world phenomenon or until now only a hypothetic scenario, it is quite obvious that forms of simulations, equipped with artificial intelligence (AI) will play an essential role in future healthcare. Currently, a lot of brilliant theoretical and ethical work has been done on better understanding of how the digital shapes and possibly transforms established structures and procedures; as for example, familiar concepts of agency, ${ }^{8}$ existing modes of trust, ${ }^{9}$ needs for transparency and explainability ${ }^{10}$ or questions whether specific forms of digital entities should have legal and/or moral rights. ${ }^{11} 12$ With the digital twins, however, the current debates will be intensified: what is at stake if an AI-driven simulation takes on forms of representation and acting on behalf of a physical person? Such digital twins do not simply interact with physical persons, but they operate on parts of the body as representatives or make predictions about future health events of the physical person.

Broadly speaking, the term 'twin' is understood to refer to a close entanglement between an object or an entire person and its or her simulation. There are some similarities to the concept of a clone, ${ }^{13}$ but when compared with each other the concept of a digital twin points to at least two distinctive features: first, it is strictly designed and produced with the idea of identifying and defining characteristic functions of the simulated object or person and using them as the basis of the simulation. Second-and of great importance for ethical reflection-the concept of a twin is not only restricted to improving diagnosis and therapy but also focused on providing predictions on future states of health or illness. Digital twins, thus, can be used to simulate parts of the body or even the whole body of a person in order to make prognoses and in particular to predict future developments or behaviours. Returning to the example of the severe heart defect, the simulation can not only be used to make a prognosis regarding the best treatment decision, but it may also be used to predict a severe defect in advance. This prediction could be adapted in relation to the simulated person's current or future lifestyle. Further, the digital twin can also assume different forms of digital embodiment and may also interact with the simulated person by 'knocking on her door' to warn her or suggest lifestyle changes.

In order to gain a better understanding of the possible ethical and societal impact of digital twins, this article starts by describing and analysing the different approaches of digital twins as well as their implications for medicine. Against the background of this analysis, it will become evident that digital 
twins can be used in very different ways. It is crucial to better understand these different ways of possible use before we will investigate how they can be used for different forms of representation. What happens to the representation of a person created through and by a simulation if it gains a visible and haptic form and thus becomes part of the physical world? In more theoretical words: the foreseeable rise of digital twins in medical contexts broadens the debate from a focus on the agency, patency or fairness of AI systems towards the possible embodiment of such simulations and the control of embodied persons on their simulated representations. I will argue that the embodiment of simulations such as those of digital twins not only presents new challenges but at the same time may represent a special opportunity for the form of control a person has over the simulation that stands or acts on behalf of.

In a first step, I will analyse different types of digital twins with a specific focus on the concrete challenges inherent in the interactions between persons and their digital twin. In a second step, I will address the question on how far a twin can and should be used to act on behalf of a person and what the consequences of this may be. In a third and last step, I will define first conditions for developing and using digital twins to take on an ethically justifiable form of representation.

\section{EVERYTHING CAN HAVE A TWIN? A SHORT GENEALOGY OF THE CONCEPT OF DIGITAL TWINS}

It is important to take into account the fact that simulation strategies regarding the human body have their roots in, as well as a strong connection to, attempts to simulate objects and tools in the engineering industry to deal with the increasing complexity of technological systems. ${ }^{14}$ In its inception, the term 'digital twin' was first used to describe the simulation of tools and machines, for example, engines or turbines. The underlying engineering approach was to provide a relatively holistic understanding not only of a single isolated object but of a complete system or an entire production process. ${ }^{15}$ Alongside swift improvements to such simulation strategies, some challenges remain, for example, the required simulation times being too long or unforeseeable errors occurring in the initial models of physical objects. ${ }^{16}$ Three important lessons can be learnt from attempts to tackle the challenges associated with these simulation strategies: first, the quality of knowledge about the prospective simulated representation is the decisive dimension needed to achieve precise models, and thus adequate and reliable simulations. Second, the quality of the models depends on the quality of the data as well as accurate data matching and analysis. Before this, however, a further step is necessary: having access to, or being provided with the relevant set of data. This is an especially important point when such simulation strategies are used in a medical context because in this case the data are not only very diverse, but several actors-especially the particular person from whom the simulation should be created-have to be willing to participate and donate their data. Third, the future persons who deal with and operate the simulated entities have to implement suitable data management and simulation monitoring systems a long time before being able to predict possible failures. ${ }^{4}$

\section{DIFFERENT SORTS AND MODES OF DIGITAL TWINS}

The term 'digital twin' in medicine is still relatively fuzzy. What can be described as common to all different approaches and understandings is that such digital twins are driven by the idea of providing a framework to analyse emerging data-driven healthcare practices and their implications for prediction, therapy, care and perhaps also enhancement. ${ }^{17}$ As Bruynseels et al have argued, such digital twin approaches in medicine follow an engineering paradigm where individual physical artefacts are paired with digital models that dynamically reflect the status of those artefacts. In the context of medicine in particular, the idea of digital twins builds on the merger of biotech and infotech: molecular readout technologies have to be systematically matched with a large set of data in order to design and create personalised virtual models and to complement them with continuously tracked health and lifestyle parameters. ${ }^{17}$ In order to create such virtual models of human organs or even entire bodies, very detailed biophysical and lifestyle information from a person over a long period of time is necessary. This may be especially pertinent where questions of digital twins are closely connected to the development of, as well as the debates on, so-called personalised medicine.

In order to provide a concise understanding of the different simulation processes and to better face and map the challenges associated with digital twins, as a first step it is necessary to develop a more detailed description. In the following, I suggest differentiating between different forms of simulation with regard to different aspects of the interaction between a physical embodied person and her simulation. On a technical level, Chinesta et al have proposed to distinguish different forms of simulated twins. ${ }^{18}$ Conceptually building on this nomenclature, it is possible to better describe different dimensions of digital twins in relation to the respective physical entity, especially in the context of medicine: embodied persons (see table 1).

The very basic idea of simulations is to emulate a physical system by one or more mathematical models in order to describe a respective structure or behaviour. A characteristic of such a simulation is that it is more or less static. Once fed with the necessary data, it offers a detailed model of the physical object or structure and does not have to be continuously updated with data. Furthermore, such a simulation approach allows for the precise determination of whether and to what degree the simulation is an adequate model of the respective physical entity.

Digital twins still can be described as simulations. But they include several additional features. Such twins are in need of permanent input of data regarding the represented structure or behaviour. They are then able to provide real-time simulation and feedback. In many cases of basic simulations there is an individual physical object which is used as a starting point to build a model. This is not necessarily the case with digital twins. They can be designed based on specific patterns in mass-collected and

Table 1 Different forms of simulations in comparison to embodied persons (source: own work)

\begin{tabular}{llll}
\hline & Basic simulations & Digital twins & Embodied persons \\
\hline Sensors & Selected singular inputs & Sensory networks & Five senses \\
Form of processing & Mathematical functions & Artificial Intelligence and machine learning & Human brain \\
Form of adaption & Static & Dynamic & Dynamic \\
Form of appearance & Model of respective entity & Embodied model of respective entity (eg, avatar, hologram) & Physical body \\
\hline
\end{tabular}


adequately curated sets of data, while leaving open the possibility for these patterns to track or correspond to a concrete physical entity. Although there is the potential to improve databased prediction as well as real-time decision making, the price to pay is a huge investment in the permanent validation of the created simulation. ${ }^{18}$ Such a validation should ensure that the embedded and utilised set of data is correctly analysed and guarantee that there is a valid correlation between the simulation and the simulated object or entity. Digital twins can further be used to combine a physically based model with a specific data pattern model for accommodating real-time feedback. Such forms of digital twins consist of three basic components: first, a simulation core that can solve complex mathematical problems representing physical models under real-time constraints; second, advanced strategies that enable data assimilation, data curation and data-driven modelling; and third, mechanisms to adapt the model to evolving environments online. ${ }^{18}$ Not least digital twins are backed by several forms of machine learning and algorithmic data processing and may in advanced forms also be equipped with something we can describe as physical embodiment-perhaps not the same sort of embodiment as an embodied person, but a visible and haptic form of representation in the physical world.

Returning to our example of the severe heart defect, speaking of digital twins can have different implications. In the case of the basic simulation, there would be an individual simulation of the heart. Such a simulation could serve as a precise model of the physical object. In the digital twin approach, there would not only be a simulated model of the heart, but this would be updated with changes regarding the condition of the physical object. It would also be integrated into a larger and more importantly a dynamic simulation of the broader environment, or even a simulation of the whole person. Such forms of simulation are able to predict possible progressions of diseases as well as suggest different treatment options based on a permanent analysis of the connected data. Under specific conditions, they become a visible part of our physical world and are embodied in a haptic form. This would perhaps not be a physical human body but a body in the sense that there is a specific physical representation of this simulated twin, for example, an avatar or a hologram. This may also imply consequences for the interaction between the human person and 'their' digital twin (see figure 1).

\section{SIMULATING A PRECISE EFFIGY}

The central question of this paper is whether, and if so to what extent, a simulated twin can be used to represent an embodied person. Representation as it is understood here means that the simulation functions as a sort of surrogate for the simulated object or entity in a specific context: in our case medicine. This is an especially important challenge as the simulation is, even under the condition of real-time simulation, not located in exactly the same time and space as the represented physical entity. Thus, the term 'representation' is focused on the specific interaction between the embodied person and her simulated twin. Accordingly, an initial necessary but not sufficient central aspect of representation is the correspondence between a person and her simulation. Furthermore, in order to think about a simulation that represents a person in a specific context, the interaction between the person and her simulation comes to the fore.

A necessary condition for representation would be a form of correspondence between the person and the simulation. The term 'correspondence' is understood to refer to the extent to which the simulation is precise and, in this sense, an adequate

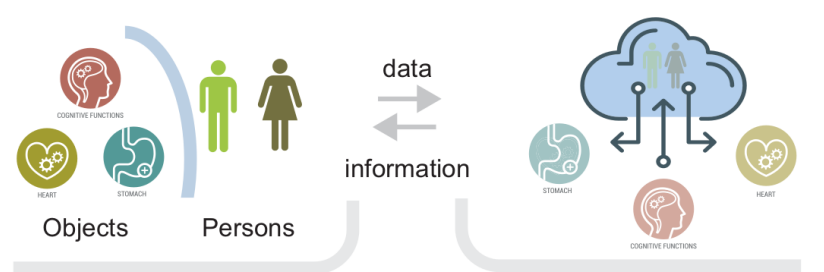

Physical Lifeworld

\section{Simulated Lifeworld}

TWINS

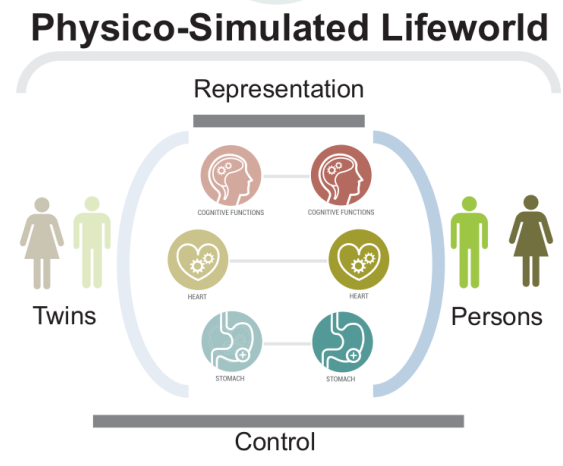

Figure 1 Shifts and transformations between physical and simulated lifeworld (source: own illustration). The conceptually interesting aspect of the simulated twin approach is that to some extent it merges the lifeworld of physically embedded persons with their respective simulated twin. The idea of letting a simulation act as a representation of a person (and perhaps a representation that is embodied) forces us to think not only about the interaction between a person and her simulated twin but-especially with regard to the twin's predictive power-about the modes of control required to maintain the direction of interaction in a way that is adaptive to personal modes of freedom.

model of the simulated object or entity, for example, the simulated virtual heart. Thus, the first central criterion for such a correspondence is whether-and if so, to what degree and under which circumstances-the simulated object is a precise effigy of the physical object or entity. ${ }^{19}$ But what does it take to be a precise effigy? A conventional answer would perhaps be that the simulation has to be based on adequate and precise data. And this, of course, is an essential point. With regard to the example of the severe heart defect, using a possible in silico model is a responsible approach only if the simulated heart is definitively an adequate simulation based on precise data, and this requires regular updates.

But at the same time, there is another entangled point here: the precision of the simulation not only depends on whether all possible data have been considered and are correctly embedded and analysed in the simulation model, but the required precision of the model itself depends on the specific context in which the simulation should be used. Thus, the specific context in this case is not merely an extrinsic issue in the sense that the simulation could be developed as an independent model and then has to be adapted to the specific context afterwards. Instead, the context is an inherent criterion for the required precision of the simulation in itself. This is especially important when we take into account that the required data in the field of the digital twin are not individual data but correlations and inferences out of large population-based data sets. In such cases, it is essential that 
the conclusions drawn about a person by means of correlation and analogies apply to the person represented. ${ }^{20}$ The respective context therefore represents a central distinguishing feature here, insofar as different contexts are associated with different degrees of vulnerability of the person to be represented. ${ }^{21} \mathrm{~A}$ prediction about the probability to get cancer within the next year has a different risk to add new injuries than, for example, a prediction about training optimisation. Although in both cases there may well be significant risks of injury to the represented person, the particular context decisively determines the time period for which these will be expected.

Second, this leads to questions of transparency: in order to determine and ensure that the simulation is adequate in the specific context, transparency is needed not only with regard to the data used but also to the standards used, as well as the background presumptions. At this point, it is important to differentiate between different users of the simulation. On the one hand and most importantly, the person whose organ or even entire body is simulated must have the right and the possibility not only to know about possible bias but also about the actual (planned) use of the simulation. On the other hand, the clinician needs transparency regarding the data included, the methods and models used as well as the quality in order to gain proof and thereby security as to what degree the simulation could still refer to evidence-based standards in medicine.

Whether a precise effigy and transparency are sufficient for correspondence depends once again on the concrete context of the simulated entity. If a relatively simple object is simulated, they may be sufficient. But in the specific clinical use of simulated twins, the simulated objects are not mere mundane objects: they are treated as if they are for example a person's organ. This means that there is a direct and fundamental dependency of the person on the correspondence between the simulated organs and the physical ones. Again, at this point, it is important to take a close look at the respective context in which a simulation is or should be used. For example, the simulation of a person has a completely different significance in the context of a sporting simulation - where a correspondence between Lionel Messi and his simulation has effects on modes of personal and social recognition-or the context of medicine where the correspondence between the simulated and the physical heart may be a question of life or death.

\section{REPRESENT ME: PLEASE!?}

Besides the necessary dimension of correspondence between a person and her simulation, a second aspect has to be analysed. To represent somebody implies a specific kind of interaction between the person and her simulation. Specific in the sense that it is not a symmetric but an asymmetric interaction between a person and a simulation-or to be more precise: her simulation. But what does it mean if a simulation is her simulation? One possibility would be to claim that the simulation of a person has to be the property of the person. This may be possible, but due to legal aspects it is difficult. Furthermore, the debate about ownership of data or simulations tends to suggest a false alternative: the question is not whether or not a person owns her simulation but if the person has the right as well as the power to direct the way in which her simulation is used. In more concrete terms, it is a question of control of the simulation and especially its use as far as the simulation is not only a parallel simulation of the respective person but under specific conditions takes the place of the person. As in the example of the severe heart defect, this could offer important advantages. If my digital twin is a very precise effigy, the simulated heart can be tested instead of the physical one. This may provide insights and open up possibilities that would not have been possible through an investigation of the physical heart. In this sense, the simulation takes the place of a person's organ in order to generate more precise knowledge about the physical heart or predict further developments before their potential manifestation in the simulated object. Still, both the simulation as well as the physical object can be described as two distinct entities. But on the level of medical practice as well as the considered actions, the simulation is conceived of as a surrogate representing the body of the represented person.

Accordingly, the simulation is neither wholly one's own nor completely foreign. ${ }^{22} 23$ This seems to be a paradox at first glance. The reason is that the person who gives power to a surrogate to represent her never remains uninvolved. On the one hand, the surrogate has to act as if she is the person represented. On the other hand, there has to be a clear distinction between the surrogate and the represented entity in order to not be replaced by the representing entity. The represented person cannot remain in the position of an allegedly uninvolved observer when the simulated twin 'acts' in her name. In this respect, as the philosopher Lévinas also showed in his remarks on representation, ${ }^{24} 25$ giving power to a simulation to act in my name implies the risk of illegitimate replacement of the person by the simulation. Substitution in this context describes a phenomenon which is also well known in cases where a real person represents another real person in medical contexts. The fundamental idea is that the representing person acts as a kind of surrogate in the name of the represented person. This enables the represented person to have a say in places where she cannot act immediately or cannot have a say anymore. For example, if a person has had a stroke or has a severe mental disorder such as dementia, she needs somebody who takes her place and acts in her name. But even if the representing person represents in the best way and tries to perfectly imagine the will of the represented person, she remains a substitute. Thus, substitution takes into account that to be represented can ultimately lead to the experience that the representing person or simulation is not acting on behalf of the represented person anymore.

The tension between owning the representing simulation and being aware that the representation is not substituting the represented entity increases even more if my simulated twin is not only a representation of me and acts in specific contexts on my behalf but is able to predict smaller or larger parts of a person's future. As an example, the digital twin simulating my cardiovascular system may one day 'knock on my door' in order to warn me of an imminent severe heart disease. In any case, this will change my life completely because besides the possibility of offering me opportunities to ward off the physical manifestation of disease, the digital twin will be exercising power over me and my life. Once again in the words of Lévinas, ${ }^{26}$ there is a remaining ambivalence in representation, which oscillates between enabling new modes of freedom and endangering freedom by being captured by the predictive power of the simulated twin. Consequently, representation and acting on behalf can be described as a process which is not reversible at will. In this sense, representation processes are always prone to be disrupted and involve a high degree of ambiguity. 
In the eyes of some scholars, for example, the French philosopher Baudrillard, this fundamental ambiguity of representation is further enhanced when not a real person but a digital simulation is acting on behalf: the '.. simulation threatens the difference between the 'true' and the 'false', the 'real' and the 'imaginary'.' This also has direct consequences for the person who is represented by a simulation. The simulation, '... the one of which we have always already dreamed (but these are only poor bricolages of it) gives us the feeling, the vertigo of passing to the other side of our own body, to the side of the double, luminous done, or dead twin that is never born in our place, and watches over us by anticipation. ${ }^{27}$ Thus, the argument-currently repeated in very different contexts-is the following: a simulation does not have the same connection to the physical world as an embodied person and thus will fundamentally endanger the freedom of the embodied person-or even substitute it. A fundamental scepticism against simulations as a legitimate representation of corporeal persons may be plausible at first glance. However, I will counter this with two arguments:

First, the Baudrillardian scepticism is not a necessary scenario. It is quite possible to limit or shorten the process of representation as long as the direction of interaction is clear and can be controlled. As Baudrillard mentioned, it is not the case that the simulation will necessarily replace the physical person. Quite the opposite is true: using a simulated twin may be a form of personal self-determination as long as the simulated person is in charge and has control of the simulation. The prerequisite for this, however, is that representation is not conceived of solely as a technical one-off act but as embedded in an ongoing relation between a person and their simulation(s). In order to deal with this ambivalence of representation, there can be good reasons why a person, be it in private or clinical representation, modifies the manner of representation, transforms it into another form, interrupts it or even ends it.

Second, there still remains a problem: if a simulation is adequate, that is, corresponds to the respective entity, and if we have faced the fundamental challenges of representation, the decisive ethical concerns the concrete challenge of control of the simulation. The line of argumentation will be that such an embodiment is not a severe problem and could be used to pave the way for a responsible handling of simulated twins. In order to better grasp this aspect, we have to take a look at what could be meant by speaking of an embodied person as being represented by an 'embodied' simulation.

\section{INTERACTING WITH AN EMBODIED SIMULATION}

Interestingly, even the body of a person is not simply present, but as the philosopher Merleau-Ponty ${ }^{28}$ put it, is at the same time there and not there. The body of a person is there insofar as it can be seen, touched, smelled or heard. But at the same time, it is not simply there either in so far as it can be disregarded, ignored or overheard. Thus, an embodied person is, so to speak, seeing and visible, hearing and audible, touching and touchable and thus eludes a clear classification of what belongs to her and what is brought to her by others. In this respect, we could follow Merleau-Ponty's theory to the idea that the body of a person is always permeated by elements of ambiguity. Seeing, hearing, feeling as well as speaking and acting persons appear simultaneously as me, we and she. The decisive point is that a person understands and can describe a body as her own body. The talk about my body then does not designate a substantial area of one's own but a relationally located preference, which designates a body as mine, yours or hers. ${ }^{29}$ This is because it is subject to a certain temporality, needs a certain place and depends on being represented by others in places where she cannot personally be at the same time. In this sense, representation by a digital twin must not be understood as an attack on the person's integrity or the conditions of her self-realisation but can be understood as a prerequisite for a person to exercise her own freedom in certain contexts. For example, an in silico operation of the digital twin can provide diagnostic insights or therapeutic opportunities, or a digital twin can be used to provide healthcare to those without access to medical facilities.

The consequence of these conceptual considerations would be that such representing simulations can best be described as further extensions of a person's body. They are equivalent to prostheses in that they are an integral part of the person's body. Just as prostheses offer new possibilities to act and participate in social life as well as to enable experiences one would not be able to have without them, the digital twin can be used to offer pathways for new modes of possible social engagement of the person as well as helping to mitigate health risks. It would be a false dichotomy to see the digital twin as a fundamental threat to the embodied person. Quite the opposite: it can be used-as a mere object or a surrogating representation-to support the vulnerable physical body. Whether the digital twin is a benefit or a threat for the individual freedom of the simulated person then depends on the concrete context of use as well as the possibility of directing and controlling the interaction with the simulation. Even here the analogy to prostheses is very helpful. The benefits as well as the risks of a prosthesis depend on whether or not it works the way the respective person wants the simulation to (in the sense that she has given her consent) and has control of it.

Yet of course, there remains a difference between a digital twin and a prosthesis-even though there are a lot of prostheses that are equipped with high tech and even parts of deep learning or AI. The first disparity is that the digital twin may create the effigy of the person by using population-based data to find patterns and correlations similar to those of the simulated person's-which would be much more complicated to control. One reason is that it is nearly impossible for the individual person to foresee the outcomes and understand the consequences of the patterns and inferences produced by the digital twin. ${ }^{30}$ The second disparity is that it is very difficult to grasp the digital twin as a part or an extension of one's own body as long as there is no visible or even haptic connection to the physical world. The reason for this lies in the embodiment of persons themselves: a person needs certain techniques and information to be able to imagine 'her' simulation at all. This is different with embodied simulations: they impose themselves, force a response and thus-as we have seen with Levinas-the possibility of direct control. ${ }^{31}$ From this perspective, the current debates on a possible agency of machines, algorithms or robots can also be seen as a debate on the bodily dimension of the interaction between humans and machines.

From this perspective, digital simulations can be part of ensuring direct interaction and control because their specific embodiment engages and fosters more direct interaction. This would be especially important in cases where not only 
separate organs are simulated but an entire body. Such an embodiment of the simulation can of course be used for problematic purposes as well. As in other contexts of representation, such surrogating processes remain ambivalent and depend on the concrete level of possible control.

\section{IN SEARCH OF POSSIBLE ORIENTATION IN DEALING WITH DIGITAL TWINS}

The rise of digital twins in the medical context offers opportunities as well as challenges. As discussed in this article, one central task is the challenge of the representation of a person by a simulation. How can it be ensured that the person is represented in a way she decides and still has the opportunity to direct the representation in a way that serves her desires and enables her self-determination?

First, it has become an important result of the present discussion that digital twins do not fundamentally threaten the embodied person, neither do they necessarily interfere with the bodily integrity of a person or pose challenges which cannot be addressed.

Second, this first observation depends on the concrete possibility of a person having control over her simulated representation. This requires dynamic consent as to whether and in which context a person would like to be represented by a digital twin. Furthermore, a person should-whenever possible — be able to choose both: the preferred form of simulation as well as its usage in terms of therapy and/or predictions on future health events.

Third, in cases where a person does not have control over her digital twin-that is, is not able to give consent or have awareness of as well as knowledge about her current representation or is not able to reject or modify consent-such simulations threaten to become illegitimate representations. They would then shift the kind of interaction from representation to illegitimate forms of prediction or surveillance and thereby could lead to infringements to individual modes of freedom. In this sense, a responsible way to deal with digital twins in the future will need a focus on interoperable interfaces between a person and her simulation. As argued in this paper, the embodiment of the machine can be a promising way to enable a person to have a concrete interaction with her representation.

Fourth, besides the questions with regard to the individual, the future rise and possible use of simulations as representations also entails questions of justice. It will be and remain an important societal question to deliberate and discuss who should and must have access to these simulated forms of representation. If-as argued in this article-representation is such a fundamental experience as well as condition of human existence, the access to good and robust representations of a person must not solely be a question of financial, societal or political power. Instead, it will be of utmost importance to societally elaborate and discuss the required decent minimum of access to the digital twins.

Fifth, one important focus in order to handle the predictive power inherent in the digital twin approaches is to look at the structures behind them-and this of course implies the economy behind them as well. The current digital twin approaches are all developed in the private economic sector driven by big tech companies with-at least recently-limited possibilities for public participation. At this point, analysing what is at stake as well as laying the foundations of an ethics of digital twins is only one part of the required ethical consideration and work. Furthermore, we have to integrate the conceptual engineering work into the technological development of the twins. In this sense, the current and future rise of digital twins may also reshape the necessary approach of ethical reflection and deliberation. The current crucial question is how we can find robust and innovative ways not only to follow the pathways of technological development but to shape the way in which such transformations will unfold.

Twitter Matthias Braun @brau_matt

Acknowledgements This work would not have been possible without the criticisms and comments on earlier versions of this article of Hannah Bleher, Peter Dabrock, Patrik Hummel, Jenny Krutzinna, Barbara Prainsack, Marit Skivenes, Max Tretter as well as the two anonymous reviewers. All possible errors are my own.

Contributors MB is the sole author of this article. MB serves as an assistant professor at the Chair of Systematic Theology II (Ethics) at Friedrich-Alexander University Erlangen-Nürnberg. His research is focused on theological, philosophical and sociopolitical concepts in dealing with emerging biotechnologies at the sciencesociety interface (with special regard to synthetic biology, stem cell research and bigdata medicine) on the one hand as well as concepts of recognition and vulnerability and its implication for ethics on the other hand.

Funding This work has been funded by a grant by the Federal Ministry of Research and Education (grant number: 01GP1905B).

Disclaimer The funder has no role in the research.

Competing interests None declared.

Patient consent for publication Not required.

Provenance and peer review Not commissioned; externally peer reviewed.

Data availability statement There are no data in this work.

Open access This is an open access article distributed in accordance with the Creative Commons Attribution Non Commercial (CC BY-NC 4.0) license, which permits others to distribute, remix, adapt, build upon this work non-commercially, and license their derivative works on different terms, provided the original work is properly cited, appropriate credit is given, any changes made indicated, and the use is non-commercial. See: http://creativecommons.org/licenses/by-nc/4.0/.

ORCID iD

Matthias Braun http://orcid.org/0000-0002-6687-6027

\section{REFERENCES}

1 Björnsson B, Borrebaeck C, Elander N, et al. Digital twins to personalize medicine. Genome Med 2020;12(1):4

2 Li X, Liu X, Wan X. Overview of digital twins application and safe development. Journal of System Simulation 2019;31(3):385-92.

3 Goh EL. How digital twins of the human body can advance healthcare, 2018Hewlett Packard enterprise. Available: https://www.hpe.com/us/en/newsroom/blog-post/2018 07/how-digital-twins-of-the-human-body-can-advance-healthcare.html

4 Armendia M, Alzaga A, Peysson F. Twin-Control Approach. In: Armendia M, ed. TwinControl: A Digital Twin Approach to Improve Machine Tools Lifecycle. Springer Nature: Cham, 2019: 23-38.

5 Botkina D, Hedlind M, Olsson B, et al. Digital twin of a cutting tool. Procedia CIRP 2018;72(3):215-8.

6 Lehrach $\mathrm{H}$, lonescu A, Benhabiles N. The future of health care: deep data, smart sensors, virtual patients and the Internet-of-Humans, 2016. https://docs.wixstatic. com/ugd/2b9f87_40d29af47a9742498cbbbd484e0174e0.pdf

7 Fernando L, Haag DS, Anderl R, et al. Digital twin requirements in the context of industry 4.0, in product lifecycle management to support industry 4.0. In: Chiabert P, ed. Product lifecycle management to support industry 4.0. Cham: Springer, 2018: 204-14

8 Nyholm S. Humans and robots: ethics, agency, and Anthropomorphism. London: Rowman \& Littlefield Publishers, 2020.

9 Ryan M. In Al we trust: ethics, artificial intelligence, and reliability. Sci Eng Ethics 2020;26(5):2749-67.

10 Coeckelbergh M. Al ethics. Cambridge: MIT Press, 2020

11 Gunkel D. Robot rights. Boston: MIT Press, 2018.

12 Danaher J. Welcoming robots into the moral circle: a defence of ethical Behaviourism. Sci Eng Ethics 2020;26(4):2023-49.

13 Papacharissi Z, ed. A Networked Self and Human Augmentics, Artificial Intelligence, Sentience. New York: Routledge, 2016.

14 Grieves M, Vickers J. Digital twin: mitigating unpredictable, undesirable emergent behavior in complex systems, in Transdisciplinary perspectives on complex systems. In: Kahlen F-J, Flumerfelt S, Alves A, eds. Transdisciplinary perspectives on complex systems. Cham: Springer, 2017: 85-113.

15 Vogt H, Hofmann B, Getz L. The new holism: P4 systems medicine and the medicalization of health and life itself. Med Health Care Philos 2016;19(2):307-23. 


\section{Feature article}

16 Tao F, Qi Q, Wang L, et al. Digital twins and Cyber-Physical systems toward smart manufacturing and industry 4.0: correlation and comparison. Engineering 2019;5(4):653-61.

17 Bruynseels K, Santoni de Sio F, van den Hoven J. Digital twins in health care: ethical implications of an emerging engineering paradigm. Front Genet 2018;9:31.

18 Chinesta F, Cueto E, Abisset-Chavanne E, et al. Virtual, digital and hybrid twins: a new paradigm in data-based engineering and engineered data. Arch Computat Methods Eng 2020;27(1):105-34.

19 Johnson E. Simulating Medical Patients and Practices: Bodies and the Construction of Valid Medical Simulators, in Technology and Medical Practice. In: Johnson E, Berner B, eds. Blood, guts and machines. Burlington, Farnham, 2010: 119-40.

20 Lupton D. 'Not the Real Me': Social Imaginaries of Personal Data Profiling. Cult Socio 2020.

21 Braun M. Vulnerable life: reflections on the relationship between Theological and philosophical ethics. Am J Bioeth 2020;20(12):21-3.

22 Barad K. Getting real: Technoscientific practices and the materialization of reality. differences. A Journal of Feminist Cultural Studies 1998;10(2):87-128.
23 Barad K. Agential Realism: How Material-Discursive Practices Matter, in Meeting the Universe Halfway. In: Barad K, ed. Quantum Physics and the Entanglement of Matter and Meaning. Durham, London: Duke University Press, 2007: 132-85.

24 Lévinas E, Nous E. Essays on Thinking-of-the-Other. Translated by Michael B. Smith and Barbara Harshav. New York: Columbia University Press, 2000.

25 Lévinas E. Other wise than Being or Beyond Essence. Translated by Alphonso Lingis. Pittsburgh: Duquesne University Press, 1998.

26 Lévinas E. The Trace of the Other. In: Taylor MC, ed. Deconstruction in context: literature and philosophy. Chicago: University of Chicago Press, 1986: 345-59.

27 Baudrillard J. Simulacra and simulation. Michigan: The University of Michigan Press, 1994.

28 Merleau-Ponty M. Phenomenology of perception. 2nd edn. London: Routledge, 2002

29 Waldenfels B. Sinnesschwellen. Studien zur Phänomenologie des Fremden. Frankfurt a.M: Suhrkamp, 1999

30 Wachter S, Mittelstadt B. A right to reasonable inferences: Re-Thinking data protection law in the age of big data and Al. Columbia Business Law Review 2019;2.

31 Heinrichs J-H. Virtual action. Ethics Inf Technol 2020;17(4). 